\section{PSICOLOGIA IBEROAMERICANA}

\section{Psicología lberoamericana}

ISSN: 1405-0943

psicología.iberoamericana@uia.mx

Universidad Iberoamericana, Ciudad de

México

México

Sánchez Bravo, Claudia; Morales Carmona, Francisco; Carreño Meléndez, Jorge; Cuenca Vázquez, José

Disfunciones Sexuales Masculinas: su Asociación con el Malestar Psicológico y los Problemas

Conyugales en una Población de la Ciudad de México

Psicología lberoamericana, vol. 15, núm. 1, junio, 2007, pp. 60-67

Universidad Iberoamericana, Ciudad de México

Distrito Federal, México

Disponible en: http://www.redalyc.org/articulo.oa?id=133915928008

Cómo citar el artículo

Número completo

- Más información del artículo

Página de la revista en redalyc.org

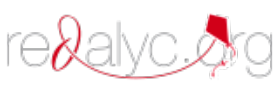

Sistema de Información Científica

Red de Revistas Científicas de América Latina, el Caribe, España y Portugal

Proyecto académico sin fines de lucro, desarrollado bajo la iniciativa de acceso abierto 


\title{
Disfunciones Sexuales Masculinas: su Asociación con el Malestar Psicológico y los Problemas Conyugales en una Población de la Ciudad de México
}

\author{
Male Sexual Dysfunctions: its Association with \\ Psychological Distress and \\ Marital Problems in a Population of Mexico City
}

\author{
Mtra. Claudia Sánchez Bravo* \\ Dr. Francisco Morales Carmona** \\ Mtro. Jorge Carreño Meléndez* \\ Lic. José Cuenca Vázquez ${ }^{* * *}$
}

\section{Resumen}

Objetivo: Evaluar la relación entre cada disfunción sexual masculina, el malestar psicológico y los problemas conyugales. Método: Estudio de campo, retrospectivo, transversal, comparativo, de una muestra dividida en dos, procedimiento de selección por cuota de los cónyuges de las pacientes del INPERIER. La muestra fue de 200 participantes: cien hombres sin y cien hombres con disfunciones sexuales. Se utilizaron el Cuestionario de sexualidad versión hombres y el Cuestionario general de salud.

Resultados y conclusiones: Hombres con deseo sexual hipoactivo y eyaculación precoz presentan un mayor malestar psicológico. Los problemas conyugales se relacionan con las disfunciones masculinas, observando la mayor relación en el trastorno del orgasmo y deseo sexual hipoactivo, lo cual es un indicador de riesgo de malestar psicológico.

Descriptores: disfunciones sexuales masculinas, malestar psicológico, problemas conyugales, indicadores de riesgo, plan de tratamiento.

\footnotetext{
Abstract

Objective: To assess the relationship between each male sexual dysfunction, psychological distress and marital problems Method: Field study, retrospective, cross-sectional, comparative of a single sample divided in two, quota selection procedure of the patients' spouses of INPERIER. The sample consisted of 200 participants: 100 men without sexual dysfunction and 100 men with sexual dysfunctions. The Questionnaire of Sexuality Male Version and the General Health Questionnaire were used.

Results and Conclusions: Men with hypoactive sexual desire and premature ejaculation show a higher psychological distress. Marital problems are related with male sexual dysfunctions, observing a greater relation in the orgasmic and hypoactive sexual desire disorder, which is a risk indicator of psychological distress.

Key words: male sexual dysfunctions, psychological distress, marital problems, risk indicators, treatment plan.

* Psicólogos adscritos al Departamento de Psicología, Instituto Nacional de Perinatología "Dr. Isidro Espinosa de los Reyes".

** Jefe del Departamento de Psicología del Instituto Nacional de Perinatología "Dr. Isidro Espinosa de los Reyes".

*** Servicio social. Universidad Iberoamericana.

Correspondencia:

Mtra. en Psic. Claudia Sánchez Bravo. Departamento de Psicología INPERIES

Montes Urales 800, Col. Lomas Virreyes, C:P: 11000, México, D.F. clausanbra@yahoo.com
} 


\section{Introducción}

El estudio de los problemas sexuales ha tomado gran relevancia, pues el desarrollo adecuado de la sexualidad a lo largo de la vida del individuo es un indicador de bienestar psicológico, de ahí que la Organización Mundial de la Salud (oms) defina la salud sexual como: "La integración de los aspectos somáticos, emocionales, intelectuales y sociales del ser humano sexual en formas que sean enriquecedoras y que realcen la personalidad, la comunicación y el amor", y señala tres condiciones para que se dé: a) capacidad para disfrutar la conducta sexual y reproductiva de acuerdo con una doble ética: social y personal; $b$ ) estar libre de temor, vergüenza, culpa y otros factores psicopatológicos que inhiben la respuesta sexual y limitan las relaciones sociosexuales, y c) estar libre de perturbaciones, enfermedades, o limitaciones orgánicas que interfieran con la salud sexual y/o reproductiva (Álvarez Gayou, 1986). De ahí que la salud sexual sea salud mental y ésta expresión de la salud integral. El interés creciente por investigar distintos aspectos de la sexualidad humana ha sido el resultado de los estudios multivariados realizados. Aunque haya nociones diferentes, según la teoría que se trate, todos coinciden en darle un significado más amplio, como un fenómeno que se refiere a todos los planos del ser humano. Hablar de sexualidad es referirse a una dimensión de la personalidad, no sólo a la aptitud del individuo para generar una respuesta erótica. El estudio concreto de la respuesta sexual humana y la definición de sus fases llevaron a describir y delimitar las alteraciones sexuales que se presentan en cada una de ellas. En la década de 1960, Masters, Johnson y Kolodny (1995), estructuraron por primera vez la respuesta sexual humana y sus disfunciones. Dicho modelo describe una secuencia ordenada de un acontecimiento unitario e inseparable, que responde a distintos niveles de excitación sexual, lo que llevó a entender el desempeño sexual del individuo con sus variantes individuales, que incluyen no sólo la fisiología sino también aspectos psicológicos y sociales. Ellos mostraron cómo se comporta el ciclo de la respuesta sexual humana, al que dividieron en cuatro fases: excitación, meseta, orgasmo y resolución. Posteriormente, agregaron -aporte sin lugar a dudas importante en este ciclo- dos fases más: la fase de deseo y la fase refractaria. Esta estructuración es una forma esquemática de conceptuar fenómenos fisiológicos para facilitar su estudio, pues el paso de fase a fase no se observa con claridad, habiendo variacio- nes de un individuo a otro, refiriendo que cuando la función sexual es normal ésta se acepta, suponiendo que tiene que ser así, sin que cause alteración alguna, pues el individuo ni siquiera piensa en ello, sin embargo cuando dicha función se convierte en un problema es fuente de angustia y frustración, lo que genera una sensación de malestar e insatisfacción en las relaciones interpersonales.

En las disfunciones sexuales, su estudio, definición y clasificación ha permitido el establecimiento de estrategias de tratamiento. Las definiciones más representativas están descritas como:

Alteraciones persistentes o recurrentes de una o varias fases de la respuesta sexual, que provocan problemas o molestias al individuo o pareja (Álvarez Gayou, 1986).

Serie de síndromes en los que los procesos eróticos de la respuesta sexual resultan no deseables para el individuo o para el grupo social y que se presentan en forma persistente o recurrente (Rubio y Díaz, 1994).

A partir de diversos estudios sobre la respuesta sexual humana y las disfunciones sexuales, se han propuesto varias clasificaciones, pero para los fines de este trabajo se eligió la del DSM-IV-TR (American Psychiatric Association, 2002), la cual refiere que las disfunciones sexuales se caracterizan por la provocación de malestar psicológico y problemas interpersonales, dos elementos cuya comprobación es el propósito de estudio de una línea de investigación de sexualidad humana surgida por la práctica clínica realizada en el Departamento de Psicología del Instituto Nacional de Perinatología (INPERIER) de la ciudad de México -institución de cuarto nivel de atención que trata los problemas relacionados con la reproducción-, y en donde se ha detectado un alto porcentaje de pacientes y parejas con problemas sexuales, cuya prevalencia, en las mujeres es de $52 \%$ y en los hombres de 38.8\% (Sánchez, Carreño, Martínez y Gómez, 2005).

Dicha prevalencia despertó la necesidad de realizar investigaciones para estudiar factores asociados a estos fenómenos y así poder tener un mayor conocimiento de la problemática sexual; se busca aportar elementos que permitan desarrollar abordajes terapéuticos más precisos. Así, estas investigaciones se deben realizar tanto en mujeres como en hombres para tener una visión completa de la sexualidad humana.

El objetivo del presente estudio es detectar si existe relación entre el malestar psicológico y los 
problemas conyugales asociados a cada una de las disfunciones sexuales masculinas en una población de la ciudad de México.

Las disfunciones sexuales masculinas son clasificadas en DSM-IV-TR (American Psychiatric Association, 2002) de la siguiente manera:

\section{Trastornos del deseo sexual}

Deseo sexual hipoactivo: disminución o ausencia de fantasías y deseos de actividad sexual de forma persistente o recurrente.

Trastorno por aversión al sexo: aversión extrema persistente o recidivante y con evitación de todos o casi todos los contactos sexuales genitales con una pareja sexual.

\section{Trastornos de la excitación sexual}

Trastorno de la erección: incapacidad persistente o recurrente para obtener o mantener una erección apropiada hasta el final de la actividad sexual.

\section{Trastornos orgásmicos}

Trastorno orgásmico masculino: ausencia persistente o recurrente del orgasmo tras una fase de excitación sexual normal.

Eyaculación precoz: eyaculación persistente y recurrente en respuesta a una estimulación sexual mínima antes, durante o poco tiempo después de la penetración, y antes de que la persona lo desee.

Cabe observar que antes de los trabajos de Masters y Johnson (1970) las alteraciones sexuales eran consideradas como síntomas pertenecientes a otras enfermedades y el modelo era unidimensional: frigidez en la mujer e impotencia en el hombre, englobándose en estos dos términos todas las alteraciones sexuales. Posteriormente, adquirieron el carácter de entidades clínicas con tratamientos específicos, apareciendo por vez primera en el Manual de diagnóstico y estadístico de los trastornos mentales de la Asociación Psiquiátrica Americana DSM III (1980), como un apartado específico.

En cuanto al malestar psicológico, éste se ha detectado a través del Cuestionario general de salud (CGS) de Goldberg. El instrumento detecta el malestar psicológico a través del registro de la presencia de síntomas que denotan sentimientos generales de ma- lestar de leves a graves (Goldberg y Williams, 1996). Uno de estos estudios se realizó en el INPERIER con el propósito de detectar la prevalencia de malestar psicológico en la población que acude a la institución para su atención médica, encontrando que en las pacientes obstétricas hay un mayor porcentaje de malestar psicológico que en las pacientes ginecológicas. El estudio sostiene que las pacientes obstétricas cursaban un embarazo de alto riesgo, lo cual se asoció al malestar psicológico (Morales, Luque y Barroso, 2002). En cuanto a la relación con algún tipo de problemas sexuales, un estudio llevado a cabo con pacientes que acudían a la práctica médica general en Londres (Nazareth, Boynton y King, 2003), indica que los hombres que recibieron un diagnóstico de disfunción sexual presentaron un mayor malestar psicológico en comparación con los hombres sin disfunción sexual; la disfunción que se asoció al nivel más alto de malestar psicológico fue el trastorno de la erección. Asimismo, este estudio muestra que los hombres con problemas sexuales reportaron en menor medida intercambio sexual reciente y satisfacción con su vida sexual.

Por otro lado, Van Lankveld y Grotjohann (2000) reportaron una comorbilidad psiquiátrica significativa en los hombres con disfunción sexual. Estos autores sostienen que los hombres con disfunción sexual presentaron por lo regular más trastornos de ansiedad actual y diagnósticos de trastornos del estado de ánimo de toda la vida, en comparación con la población masculina general.

En una investigación se estudiaron factores sociales, psicológicos y físicos asociados con los problemas sexuales (Dunn, Croft y Hackett, 1999), encontrando una asociación entre las dificultades psicológicas y algunas disfunciones sexuales masculinas; la eyaculación precoz se relacionó con la ansiedad, mientras que la inhibición del placer se asoció con la depresión.

Dentro de los estudios predictivos, Young, Denny, Luquis y Young (1998) proponen un modelo para predecir el grado de satisfacción sexual en hombres y mujeres casados: la satisfacción general con el matrimonio en aspectos no sexuales de la relación; el tener orgasmo propio y del cónyuge; la frecuencia de actividad sexual, y la desinhibición con la pareja. Según éste modelo, la satisfacción general con el matrimonio fue el factor de más peso para predecir la satisfacción sexual. En este sentido, el vínculo marital y la solución adecuada a las vicisitudes que puedan presentarse dentro del matrimonio se encuentran asociadas con la satisfacción sexual de los cónyuges. No 
obstante, en otro estudio muestran que la interacción entre los problemas conyugales y la problemática sexual no siempre es clara y se trata de una interdependencia compleja (Hartman, 1980).

En un estudio con parejas que acudieron a una clínica para problemas maritales y sexuales, encontraron que la disfunción sexual masculina se relaciona con la percepción de un estado marital pobre, tanto para el hombre como para la mujer; la eyaculación precoz se relacionó con la visión negativa del hombre sobre el matrimonio, mientras que el trastorno de la erección se asoció con la percepción de un matrimonio pobre en ambos miembros de la pareja. En este estudio se concluye que el conflicto conyugal y la disfunción sexual se encuentran relacionados, esta relación es especialmente fuerte para el hombre (Rust, Golombok y Collier, 1988). Por el contrario, se ha encontrado que la relación entre problemas sexuales y problemas conyugales es especialmente significativa para las mujeres pero no para los hombres (Dunn, et al., 1999).

Otro estudio con parejas referidas a tratamiento familiar, sugiere que un buen vínculo sexual no es un componente esencial para un matrimonio satisfactorio, afirmando que las dificultades sexuales pueden presentarse en una relación marital funcional, además de que una vida sexual satisfactoria con la pareja no imposibilita la presencia de problemas en otras áreas de la relación conyugal (Hartman 1980).

\section{Método}

La investigación se realizó en el Departamento de Psicología del INPERIER. Se realizó un estudio no experimental, de campo, retrospectivo y transversal con un diseño comparativo, de una sola muestra dividida en dos grupos y de observaciones independientes, con un procedimiento de selección por cuota capturada de los cónyuges de las pacientes que son aceptadas por primera vez en la Institución.

La muestra estuvo constituida por 200 participantes varones y fue dividida en dos grupos: cien hombres que no presentaron disfunción sexual (grupo control o grupo 1) y cien hombres que presentaron disfunción sexual (grupo de casos o grupo 2). Los criterios de inclusión fueron: participantes masculinos (que acompañan a sus parejas a la institución para ser estudiados o no), el rango de edad fue de 25 a 45 años, con educación mínima de primaria para comprender correctamente los instrumentos de medición y con una relación de pareja de por lo menos un año. Los criterios de exclusión fueron: que no presentaran un diagnóstico previo de retardo mental o trastorno psicótico, ni alguna enfermedad orgánica que pudiera condicionar la disfunción sexual, tales como síndromes neurológicos, endócrinos, cardiopatías, problemas vasculares e infecciones genitales. La inclusión a uno u otro grupo fue determinada por la presencia o ausencia de disfunción sexual registrada por las respuestas de los participantes al Cuestionario de sexualidad versión para hombres (Morales, Pimentel y Aranda, 1998). El cuestionario fue diseñado en el INPERIER para detectar disfunciones sexuales según los criterios del Manual Diagnóstico y Estadístico de los Trastornos Mentales DSM-IV-TR (2002), el cual también explora la presencia de problemas conyugales; otro instrumento utilizado fue el Cuestionario General de Salud de Goldberg en versión de 30 reactivos (CGS-30), instrumento diseñado para detectar el estado psicológico tanto en ámbitos comunitarios como en medios clínicos de carácter no psiquiátrico; es una prueba de tamizaje. El cuestionario mide dos tipos principales de fenómenos: la incapacidad para realizar actividades saludables normales y la aparición de nuevos fenómenos reconocidos como malestar psíquico (Goldberg y Williams, 1996). El punto de corte puede determinarse si una persona es "probable caso", con los síntomas suficientes como para requerir atención psicológica, o "no caso" (González, Morales y Gutiérrez, 1992). El punto de corte es de 7/8, una puntuación por encima o igual a 8 equivale a "probable caso" y de 7 o menos a "no caso". El cGs-30 se ha utilizado eficazmente en población mexicana (MedinaMora, Padilla, Campillo, et al., 1983). Los cuestionarios fueron aplicados por psicólogos previamente capacitados. A los participantes se les invitó a participar en la investigación de manera voluntaria y se les proporcionó la carta de consentimiento informado solicitada por el Comité de Ética de la Institución.

En cuanto al análisis estadístico, se obtuvieron las medidas de tendencia central para la descripción de la muestra. Los factores sociodemográficos controlados fueron: edad, estado civil y escolaridad; para el análisis de las variables estudiadas se utilizó estadística inferencial; para establecer la presencia o no del malestar psicológico se compararon los dos grupos, posteriormente se hizo el análisis con cada una de las disfunciones sexuales, para lo cual se igualaron las muestras tomando de manera aleatoria 50 participantes del grupo 1, lo que evitó que la diferencia en el número de participantes entre un grupo 
y los demás sesgara los resultados; para la comparación de medias se aplicó una $t$ de Student, y para el análisis de los problemas conyugales una chi2 y V de Cramér, lo que permitió establecer la relación y fuerza de la misma entre los grupos y cada una de las disfunciones sexuales. El estudio se realizó con el programa estadístico spss para Windows, versión 10.

\section{Resultados}

La muestra quedó constituida por cien hombres sin disfunciones sexuales considerados como grupo $1 \mathrm{y}$ cien hombres con disfunciones sexuales, considerados como grupo 2. Los rangos de edad del grupo 1 fueron de 22 a 43 años con un promedio de 32 años \pm 4.97 y de 23 a 45 años en el grupo 2, con un promedio de 33 años \pm 5.20 . En relación al estado civil, en el grupo 1 el $80 \%$ de los participantes refirió estar casado y $20 \%$ en unión libre. En cuanto al grupo 2 , $78 \%$ se encontraba casado, $21 \%$ en unión libre y un solo participante era soltero. El promedio de escolaridad fue para el grupo 1 de $11.71 \pm 2.88$ y para el grupo 2 de 11.06 años \pm 3.33 .

Los resultados de la frecuencia de las disfunciones sexuales presentadas en el grupo 2 se distribuyeron de la siguiente manera: los 100 participantes estudiados presentaron un total de 157 disfunciones, o sea un promedio de 1.57 disfunciones por hombre, de los cuales 56 tuvieron una disfunción sexual, 33 presentaron dos disfunciones sexuales, 10 tuvieron tres y uno presentó las cinco, siendo las más frecuentes deseo sexual hipoactivo y eyaculación precoz, y la menos frecuente aversión al sexo (tabla 1).

\section{Tabla 1. Frecuencia de disfunciones sexuales} masculinas en el grupo $2(n=100)$

\begin{tabular}{|l|r|}
\hline Deseo sexual hipoactivo & 55 \\
Aversión al sexo & 5 \\
Trastorno de la erección & 34 \\
Trastorno del orgasmo & 15 \\
Eyaculación precoz & 48 \\
\hline
\end{tabular}

En cuanto a los resultados obtenidos en el análisis de la comparación de los grupos, se encontraron diferencias significativas entre un grupo y otro, con mayor presencia de malestar psicológico en el grupo de hombres con disfunciones sexuales en comparación con los hombres sin disfunciones sexuales (tabla 2).
En el análisis de cada una de las disfunciones sexuales y el malestar psicológico, los resultados fueron los siguientes:

Los hombres con deseo sexual hipoactivo presentan puntajes significativamente mayores en comparación a los hombres que no presentan ninguna disfunción sexual. En cuanto al trastorno de la erección y el trastorno del orgasmo, no se encontraron diferencias significativas entre los grupos. En eyaculación precoz, los resultados obtenidos muestran que hay diferencias significativas entre los grupos, reportando un mayor puntaje de malestar psicológico en el grupo con esta disfunción sexual. El análisis de la aversión al sexo se omitió por no cumplir los requisitos mínimos estadísticos.

En el análisis de los grupos y de problemas conyugales, los resultados muestran una asociación entre la presencia de disfunciones sexuales y los problemas conyugales, lo que indica que este factor interviene significativamente en la presencia de disfunciones sexuales. En la relación con cada una de las disfunciones sexuales y los problemas conyugales, se encontró una relación significativa con todas las disfunciones masculinas, lo que indica que este factor interviene en la presencia de cada una de ellas, observando la relación más fuerte en el trastorno del orgasmo, seguida del deseo sexual hipoactivo (tablas 4 y 5$)$.

\section{Discusión}

El estudio reveló que los hombres con disfunción sexual presentan una mayor presencia de malestar psicológico en comparación con los hombres sin disfunción sexual. Los resultados concuerdan con los encontrados en una población inglesa (Nazareth, et al., 2003); sin embargo, en el análisis realizado con cada una de las disfunciones sexuales es en el trastorno de la erección y en el trastorno del orgasmo, donde no se encontraron diferencias significativas en cuanto a la presencia de malestar psicológico, lo que difiere con este autor, que encontró la relación más alta de malestar psicológico en el trastorno de la erección. En cuanto a deseo sexual hipoactivo y eyaculación precoz, se observa que el malestar psicológico sí es un indicador relacionado con estas dos disfunciones, lo que contrasta con el estudio de Van Lankveld y Grotjohann (2000), que reportan una comorbilidad psiquiátrica significativa en los hombres con disfunción sexual. Podemos señalar que la presencia de 
Tabla 2. Descripción del malestar psicológico de los grupos estudiados

\begin{tabular}{|cccc|}
\hline Variable & $\begin{array}{c}\text { Grupo 1 } \\
\text { Sin disfunción sexual } \\
(n=100) \\
\text { Media } \pm D S\end{array}$ & $\begin{array}{c}\text { Grupo 2 } \\
\text { Con disfunción sexual } \\
(n=100) \\
\text { Media } \pm D S\end{array}$ & Valor $t$ \\
\hline CGS & $2.60 \pm 3.88$ & $4.44 \pm 5.99$ & -2.578 \\
\hline
\end{tabular}

$* \mathrm{p}=.05 * * \mathrm{p}=.01 * * * \mathrm{p}=.001$

Tabla 3. Descripción del malestar psicológico en cada una de las disfunciones sexuales

\begin{tabular}{|c|c|c|c|c|}
\hline $\begin{array}{l}\text { Disfunciones } \\
\quad \text { sexuales } \\
\text { masculinas }\end{array}$ & $\begin{array}{c}\text { Grupo } 1 \\
\text { Sin disfunción sexual } \\
\text { Media } \pm D S\end{array}$ & $\begin{array}{c}\text { Grupo } 2 \\
\text { Con disfunción sexual } \\
\text { Media } \pm D S\end{array}$ & Valor $t$ & Sig. \\
\hline Deseo sexual hipoactivo & $(\mathrm{n}=50)$ & $(\mathrm{n}=55)$ & -2.632 & $.010 * *$ \\
\hline & $2.60 \pm 4.05$ & $5.45 \pm 6.83$ & & \\
\hline Trastorno de la erección & $\begin{array}{l}(\mathrm{n}=50) \\
2.60 \pm 4.05\end{array}$ & $\begin{array}{l}(\mathrm{n}=34) \\
4.97 \pm 6.42\end{array}$ & -1.908 & $.062 \mathrm{NP}$ \\
\hline Trastorno del orgasmo & $\begin{array}{l}(\mathrm{n}=50) \\
2.60 \pm 4.05\end{array}$ & $\begin{array}{c}(\mathrm{n}=15) \\
4.87 \pm 5.30\end{array}$ & -1.766 & $.082 \mathrm{NP}$ \\
\hline Eyaculación precoz & $\begin{array}{l}(\mathrm{n}=50) \\
2.60 \pm 4.05\end{array}$ & $\begin{array}{c}(\mathrm{n}=48) \\
4.71 \pm 5.98\end{array}$ & -2.034 & $.045^{*}$ \\
\hline
\end{tabular}

$* \mathrm{p}=.05 * * \mathrm{p}=.01 * * * \mathrm{p}=.001$

Tabla 4. Descripción de los problemas conyugales de los grupos estudiados

\begin{tabular}{|lccc|}
\hline Problemas conyugales & $\begin{array}{c}\text { Grupo } 1 \\
\text { Sin disfunción sexual } \\
(n=100)\end{array}$ & $\begin{array}{c}\text { Grupo 2 } \\
\text { Con disfunción sexual } \\
(n=100)\end{array}$ & $X^{2}$ \\
\hline Si presenta & 11 & 35 & $16.262 \quad .000 * * *$ \\
No presenta & & & 65 \\
\hline
\end{tabular}

disfunción sexual masculina es un indicador de problema psicológico no psiquiátrico, que requiere de atención psicológica, sobre todo si está asociada a problemas conyugales. Para tener una mayor especificidad en el manejo de cada una de las disfunciones sexuales, se requiere del estudio de otros factores relacionados a cada una de ellas para poder tener mayores indicadores que abran la posibilidad de mejores estrategias de tratamiento.

Estos resultados parecen sustentar la idea de que la satisfacción de una persona con su matrimonio, asociada con una vida sexual satisfactoria, tiende a ser un factor central en el bienestar de una persona, coincidiendo esto con un estudio de Young, Denny, Luquis y Young (1998).

Es posible que la presencia de disfunción sexual en el hombre provoque un conflicto marital y que éste, a su vez, mantenga o empeore la dificultad sexual en el varón. Otra posible explicación consiste en que los hombres se vean considerablemente afectados por la existencia de problemas conyugales y desarrollen, en consecuencia, una disfunción sexual que impacte, a su vez, en el conflicto marital, conformándose un círculo vicioso. Se ha men- 
Tabla 5. Descripción de los problemas conyugales y cada una de las disfunciones sexuales

\begin{tabular}{|c|c|c|c|c|c|c|}
\hline \multirow{2}{*}{\multicolumn{2}{|c|}{ Disfunciones sexuales masculinas }} & \multicolumn{2}{|c|}{ Problemas conyugales } & \multirow[t]{2}{*}{$x^{2}$} & \multirow[t]{2}{*}{ Sig. } & \multirow[t]{2}{*}{$V$ de Cramér } \\
\hline & & Sí reportó & No reportó & & & \\
\hline Deseo sexual hipoactivo & $\begin{array}{l}\text { Grupo } 1 \\
\text { Sin disfunción } \\
\text { sexual } \\
(\mathrm{n}=50) \\
\text { Grupo } 2 \\
\text { Con disfunción } \\
\text { sexual } \\
(\mathrm{n}=55)\end{array}$ & 18 & 46 & 9.669 & $.002 * *$ & $.303 * *$ \\
\hline Trastorno de la erección & $\begin{array}{l}\text { Grupo } 1 \\
\text { Sin disfunción } \\
\text { sexual } \\
(\mathrm{n}=50) \\
\text { Grupo } 2 \\
\text { Con disfunción } \\
\text { sexual } \\
(\mathrm{n}=34)\end{array}$ & 11 & 43 & 4.049 & $.042 *$ & $.220 *$ \\
\hline Trastorno del orgasmo & $\begin{array}{l}\text { Grupo } 1 \\
\text { Sin disfunción } \\
\text { sexual } \\
(\mathrm{n}=50) \\
\text { Grupo } 2 \\
\text { Con disfunción } \\
\text { sexual } \\
(\mathrm{n}=15)\end{array}$ & 9 & 43 & 13.15 & $.001 * * *$ & $.450 * * *$ \\
\hline Eyaculación precoz & $\begin{array}{l}\text { Grupo } 1 \\
\text { Sin disfunción } \\
\text { sexual } \\
(\mathrm{n}=50) \\
\text { Grupo } 2 \\
\text { Con disfunción } \\
\text { sexual } \\
(\mathrm{n}=48)\end{array}$ & 18 & 43 & 7.117 & $.010^{* *}$ & $.269 * *$ \\
\hline
\end{tabular}

$* \mathrm{p}=.05 * * \mathrm{p}=.01 * * * \mathrm{p}=.001$

cionado que la interacción entre los problemas conyugales y las disfunciones sexuales es compleja y no siempre clara (Hartman, 1980).

\section{Conclusiones}

De acuerdo con el objetivo general del presente estudio, los resultados obtenidos permiten identificar algunos factores asociados a la presencia de disfunciones sexuales masculinas. El grupo de hombres con disfunción sexual muestra un mayor nivel de malestar psicológico en comparación con los hombres sin disfunción. No obstante, el grupo de hombres con disfunción sexual no rebasa el punto de corte establecido para que sea considerado como "probable caso" que requiere de atención psicológica. Con esto se sostiene que es la combi- nación de factores, en este caso disfunción sexual, problemas conyugales y/o malestar psicológico lo que requiere de intervención psicológica y que es comprobada en el presente estudio.

Esto refuerza lo detectado en el manejo de pacientes con disfunción sexual atendidos en el Departamento de Psicología del INPERIER, a lo largo de más de diez años, donde observamos en el tratamiento de pacientes que demandan atención, los casos en que se encuentra la disfunción sexual como problema único y no complicado con problemas conyugales, la terapia sexual funciona bastante bien, pero cuando se complica o es el resultado de problemas conyugales debe elaborarse un plan de tratamiento integral para el abordaje, primero de los problemas conyugales y, posteriormente y de manera complementaria, dar la terapia sexual. 
La identificación de malestar psicológico y problemas conyugales asociados a las disfunciones sexuales masculinas, enriquece el cuerpo de conocimiento existente sobre la problemática sexual. Asimismo, ofrece la posibilidad de detectar de manera oportuna la presencia de disfunciones sexuales masculinas e intervenir con mayor claridad y especificidad recibiendo un manejo especializado por los servicios de psicología correspondientes. Del mismo modo, la asociación con los problemas conyugales apunta a que la intervención debe abarcar también a la pareja, con el objetivo de aminorar y solucionar la problemática relacionada con este fenómeno, y continuar investi- gando en este campo para implementar programas de prevención y tratamiento discriminados.

Las disfunciones sexuales, el malestar psicológico y el reporte de problemas conyugales detectado o medido mediante cuestionarios o instrumentos de tamizaje, tendrían que ser criterios suficientes y válidos para recomendar tratamiento psicológico, si bien es cierto que estos aspectos se pueden presentar de moderados a graves y que pueden o no poner en riesgo la integridad de la persona, esto debe ser determinado por el psicólogo especialista, pues estas alteraciones psicológicas impactan su entorno inmediato, lo que se verá reflejado en su calidad de vida.

\section{Referencias}

Álvarez-Gayou, J. L., Sánchez, D. y Delfín, L. (1986). Sexoterapia integral. México: Manual Moderno.

American Psychiatric Association. (1980). Manual diagnóstico y estadístico de los trastornos mentales. (DSM-III). Madrid: Masson.

American Psychiatric Association. (2002). Manual diagnóstico y estadístico de los trastornos mentales. (DSM-IV-TR). Madrid: Masson.

Dunn, K. M., Croft, P. R. y Hackett, G. I. (1999). Association of sexual problems with social, psychological, and physical problems in men and women: a cross sectional population survey. Journal of Epidemiology \& Community Health, 53, 144-148.

Goldberg, D. y Williams, P. (1996). Cuestionario de Salud General. Guía para el usuario de las distintas versiones. (C. de la Cámara y S. Carreras, Trads.). Barcelona: Masson. (Trabajo original publicado en 1988).

González, C., Morales, F. y Gutiérrez, E. (1992). Indicadores clínicos de malestar psicológico en pacientes obstétricas y ginecológicas. Un estudio comparativo en México. Psicopatología, 12 (4), 147-152.

Hartman, L. M. (1980). The interface between sexual dysfunction and marital conflict. American Journal of Psychiatry, 137, 576-579.

Masters, W. y Johnson, V. (1970). Human sexual inadequacy. Boston: Little, Brown y Co.

Masters, W., Johnson, V. y Kolodny, R. (1995). La sexualidad humana. Barcelona: Grijalbo.

Medina-Mora, M. E., Padilla, G. P., Campillo-Serrano, C., Mas, C., C., Ezbán, M., Caraveo, J. y Corona, J. (1983). The factor structure of the GHQ: A scaled version for a hospital's general practice service in Mexico. Psychological Medicine, 13, 355-361.

Morales, F., Luque, M. y Barroso, J. (2002). Alteraciones emocionales en una muestra de mujeres mexicanas con eventos gineco-obstétricos. Ginecología y Obstetricia de México, 4, 157-62.

Morales, F., Pimentel, d. y Aranda, C. (1998). Sexualidad en pacientes con cáncer ginecológico. Perinatología y Reproducción Humana, 12, 170-180.

Nazareth, I., Boynton, P. y King, M. (2003). Problems with sexual function in people attending London general practitioners: cross sectional study. BMJ, 327 (7412), 423426.

Rubio, E. y Díaz, J. (1994). Las disfunciones sexuales. En Conapo (Ed.), Antología de la sexualidad humana (tomo 3) (pp. 17-46). México: Miguel Ángel Porrúa.

Rust, J., Golombok, S. y Collier, J. (1988). Marital problems and sexual dysfunction: How are they related? British Journal of Psychiatry, 152, 629-631.

Sánchez, C., Carreño, J., Martínez, S. y Gómez. M. A. (2005). Disfunciones sexuales femeninas y masculinas: Comparación de género en una muestra de la Ciudad de México. Revista de Salud Mental, 28 (4), 74-80.

Van Lankveld, J. J. D. M. y Grotjohann, Y. (2000). Psychiatric comorbidity in heterosexual couples with sexual dysfunction assessed with the Composite International Diagnostic Interview. Archives of Sexual Behavior, 29, 479-498.

Young, M., Denny, G., Luquis, R. y Young, T. (1998). Correlates of sexual satisfaction in marriage. The Canadian Journal of Human Sexuality, 7, 115-127. 